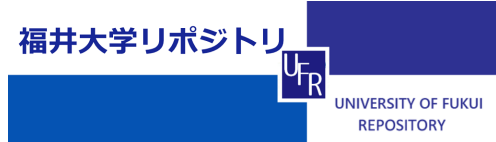

Thr ee- di mensi onal T-st resses for thr ee- poi nt-bend speci mens wi th I arge thi ckness vari at $i$ on

\begin{tabular}{|l|l|}
\hline 著者 & Lu Kai , Meshi i Toshi yuki \\
\hline $\begin{array}{l}\text { j our nal or } \\
\text { publ i cat i on t i t l e }\end{array}$ & Engi neer i ng Fract ure Mechani cs \\
\hline vol une & 116 \\
\hline page range & $197-203$ \\
\hline year & 2014 \\
\hline URL & ht t p: //hdl . handl e. net /10098/8209 \\
\hline
\end{tabular}




\title{
Three-dimensional $T$-stresses for three-point-bend specimens with large thickness variation
}

\author{
Kai Lu ${ }^{1 *}$, Toshiyuki Meshii ${ }^{2}$ \\ ${ }^{1}$ Graduate School of Engineering, University of Fukui, 3-9-1 Bunkyo, Fukui, Fukui, Japan \\ ${ }^{2}$ Faculty of Engineering, University of Fukui, 3-9-1 Bunkyo, Fukui, Fukui, Japan \\ * Corresponding author: kai_lu@u-fukui.ac.jp FAX :+81-776-27-9764
}

\begin{abstract}
Three-point-bend (3PB) test specimens are useful for the systematic investigation of the influence of statistical and constraint loss size effects on the cleavage fracture toughness of a material in the ductile-to-brittle transition temperature range. Because the in- and out-of-plane elastic $T$-stresses $\left(T_{11}\right.$ and $\left.T_{33}\right)$ are a measure of the crack-tip constraint and even the in-plane $T_{11}$ exhibits three-dimensional (3D) effects, the 3D $T$-stresses solutions were obtained by running finite element analyses (FEA) for 3PB specimens with wide ranges of the crack depth-to-width ratio ( $a / W=0.2$ to $0.8)$ and the specimen thickness-to-width ratio $(B / W=0.1$ to 40$)$. The results show that the $3 \mathrm{D} T_{11}$ at the specimen mid-plane tended to deviate from the $2 \mathrm{D} T_{11}$ as $B / W$ increased, with the deviation saturating for $B / W \geq 2$. The mid-plane $T_{33}$ increased with $B / W$ and was close to the plane strain value $T_{11}$ for $B / W \geq 2$.
\end{abstract}

Keywords: Elastic $T$-stress, Three-point-bend specimen, Finite element analysis, Fracture toughness, Constraint effect

\footnotetext{
Nomenclature

B Specimen thickness

E $\quad$ Young's modulus

F Unit magnitude (see Eq. (2))

I Interaction integral

$K_{\mathrm{I}} \quad$ Local mode I stress intensity factor (SIF)

$K_{0} \quad$ 2D SIF for elastic analysis

$R_{\mathrm{S}} \quad$ Crack tube radius

$S \quad$ Support span for 3PB specimen

$T_{11}, T_{33} \quad T$-stresses
} 


$\begin{array}{lc}W & \text { Specimen width } \\ a & \text { Crack length } \\ r, \theta & \text { In-plane polar coordinates } \\ x_{j} & \text { Crack-tip local coordinates }(j=1,2,3) \\ \Delta & \text { Singular element size } \\ \beta_{1}, \beta_{3} & \text { Normalized } T \text {-stresses } \\ \xi_{3} & \text { Out-of-plane strain } \\ v & \text { Poisson's ratio } \\ \sigma & \text { Stress components }(i, j=1,2,3)\end{array}$

\section{Introduction}

Three-point-bend (3PB) test specimens are useful for the systematic investigation of the statistical and constraint loss size effects on the cleavage fracture toughness of a material in the ductile-to-brittle transition temperature range $[1,2]$. Because the in-plane and out-of-plane $T$-stresses $\left(T_{11}\right.$ and $\left.T_{33}\right)$ are a measure of the crack-tip constraint and even the in-plane $T_{11}$ exhibits threedimensional (3D) effects [2-4], the 3D $T$-stresses solutions were obtained by running finite element analyses (FEA) for 3PB specimens with wide ranges of the crack depth-to-width ratio $(a / W=0.2$ to $0.8)$ and the specimen thickness-to-width ratio $(B / W=0.1$ to 40$)$. The $2 \mathrm{D} T_{11}$ solutions have been provided for 3PB specimen in many numerical studies [5-10].

The results show that the $3 \mathrm{D} T_{11}$ at the specimen mid-plane tended to deviate from the $2 \mathrm{D} T_{11}$ as $B / W$ increased, with the deviation saturating for $B / W \geq 2$. The mid-plane $3 \mathrm{D} T_{11}$ for $B / W=0.1$ to 40 was high as $54 \%$ when $a / W=0.2$, suggesting that $3 \mathrm{D}$ effects should be properly considered for cases of short crack length, especially when $T_{11}$ is negative. The mid-plane $T_{33}$ increased with $B / W$ and was close to the plane strain value $T_{11}$ for $B / W \geq 2$. 


\section{2. $T$-stress}

In an isotropic linear elastic body containing a crack subjected to symmetric (mode I) loading, the Williams series expansion [11] of the 3D stress components near the crack tip field can be written as [3]

$$
\left\{\begin{array}{l}
\sigma_{11} \\
\sigma_{22} \\
\sigma_{33} \\
\tau_{12} \\
\tau_{23} \\
\tau_{31}
\end{array}\right\}=\frac{K_{\mathrm{I}}}{\sqrt{2 \pi r}}\left\{\begin{array}{c}
\cos \frac{\theta}{2}\left(1-\sin \frac{\theta}{2} \sin \frac{3 \theta}{2}\right) \\
\cos \frac{\theta}{2}\left(1+\sin \frac{\theta}{2} \sin \frac{3 \theta}{2}\right) \\
2 v \cos \frac{\theta}{2} \\
\sin \frac{\theta}{2} \cos \frac{\theta}{2} \cos \frac{3 \theta}{2} \\
0 \\
0
\end{array}\right\}+\left\{\begin{array}{c}
T_{11} \\
0 \\
T_{33} \\
0 \\
0 \\
0
\end{array}\right\}
$$

where $r$ and $\theta$ are the in-plane polar coordinates of the plane normal to the crack front shown in Fig. $1, K_{\mathrm{I}}$ is the local mode I stress intensity factor (SIF) and vis Poisson's ratio. Here, $x_{1}$ is the direction formed by the intersection of the plane normal to the crack front and the plane tangential to the crack plane. $T_{11}$ and $T_{33}$ are the amplitudes of the second-order terms in the three-dimensional series expansions of the crack front stress field in the $x_{1}$ and $x_{3}$ directions, respectively.

Different methods have been applied to compute the elastic $T$-stress for test specimens, as summarized by Sherry et al. [10]. In this study, an efficient finite element method developed by Nakamura and Parks [11] based on an interaction integral was used to determine the elastic $T$ stresses.

The crack tip $T_{11}$-stress on the crack front is related to the interaction integral by

$$
T_{11}=\frac{E}{1-v^{2}}\left\{\frac{I}{F}+v \varepsilon_{33}\right\}
$$

where $E$ is Young's modulus, vis Poisson's ratio and $\xi_{3}$ identifies the out-of-plane strain at the crack tip in the direction tangential to the crack front. $I$ represents the interaction integral, and $F$ indicates the unit magnitude $(F=1)$.

Once the $T_{11}$-stress is obtained, the $T_{33}$-stress can be obtained using the following relationship:

$T_{33}=E \varepsilon_{33} \dashv v T_{11}$

More details of this method can be found in Nakamura and Parks [11] and Qu and Wang [12].

\section{Finite Element Analysis (FEA)}

3.1 Description of the finite element model

In the present study, 3D elastic FEA was conducted to calculate the elastic $T$-stresses ( $T_{11}$ and $T_{33}$ ) for a $3 \mathrm{~PB}$ test specimen with a straight crack. Fig. 2 shows a sketch of the loads and geometry. In this figure, $a, B, W$ and $S$ are the crack length and the specimen thickness, width and support span, 
respectively. For all current calculations, the specimen width was set as $W=25 \mathrm{~mm}$, with a support span of $S=4 W$.

To systematically quantify the out-of-plane crack-tip constraint effect of the 3PB specimen, the thickness-to-width ratios $B / W=0.1,0.25,0.5,1,1.5,2$ and 40 were considered to cover the $B / W$ range studied experimentally by Rathbun et al. [1]. For each $B / W$, the crack depth-to-width ratios $a / W=0.2,0.3,0.4,0.45,0.5,0.55,0.6,0.7$ and 0.8 were considered to investigate the in-plane constraint.

The material is assumed to be linearly elastic (isotropic and homogeneous). Young's modulus $E$ $=206 \mathrm{GPa}$ and Poisson's ratio $v=0.3$ were set based on ferritic steel, which is the most widely used material in engineering. 3D finite elements were used to build a one-quarter symmetric model of the 3PB specimen, as shown in Fig. 3(a). The finite element model used 20-noded isoparametric 3D solid elements with reduced $(2 \times 2 \times 2)$ Gauss integration. Sixteen singular elements were used around the crack tip for all cases in this study. Twenty equivalent rows of meshes were spaced inside the crack tube with radius $R_{\mathrm{s}}=0.4 \mathrm{~mm}$ (Fig. 3(b)). In the present FEA models, 365740 to 393194 nodes with 86912 to 93840 elements were used, and the details for the generated mesh are summarized in the Appendix.

WARP3D [13] was used as the FEA solver. The load set for the elastic FEA corresponded to the 2D SIF $K_{0}=1 \mathrm{MPa} \mathrm{m}{ }^{1 / 2}$ calculated from the following equation from the ASTM standard [14].

$K=\frac{P S}{B W^{3 / 2}} f(a / W)$

where $f$ is a function of $a / W$ and is defined in the standard.

\section{2 $T$-stresses for 3PB specimens}

$T_{11}$ was evaluated as the average of the values of $T_{11}$ obtained from domain 2 to domain 20. Good independence of the $T$ value on the choice of domain was obtained, as the differences in the $T$ stress results from domain 2 to domain 20 were within $1 \%$ of one another, except for the values in the vicinity of the free surface. The obtained mid-plane $T_{11}$ and $T_{33}$ stresses are summarized in Tables 1 and 2, respectively, in the normalized form of $\beta_{k}=T_{k k}(\mathbb{a})^{1 / 2} / K_{0}(k=1$ or 3$)$. The $T$-stresses at the specimen mid-plane received special attention because fracture initiation occurs at this location (e.g., $[1,2])$.

First, the obtained mid-plane $\beta_{1}$ values were compared with the $2 \mathrm{D} \beta_{1}$ solutions obtained by different authors [5-9] as a validity check. Sherry et al. reported that these 2D solutions varied significantly [10] and compiled them as a polynomial function of $a / W$. However, in this work, Kfouri's plane-strain solutions [6] were chosen for comparison with our 3D $\beta_{1}$ solutions based on the expectation that the $3 \mathrm{D} \beta_{1}$ will approach the plane-strain values, as shown in Fig. 4(a). The midplane $\beta_{1}$ exhibited 3D effects and monotonously decreased with increasing $B / W$ but saturated to 
values very close to the plane-strain solutions, as shown in Fig. 4(b). This tendency was similar to that observed by Nakamura and Parks for a single edge-cracked plate under pure bending [3].

Another finding was that $\beta_{1}$ was a monotonously increasing function of $a / W$, regardless of $B / W$. The results showed that negative $\beta_{1}$, and thus loss of the in-plane crack-tip constraint, was anticipated for cases of $a / W \leq 0.3$.

Fig. 5 shows the mid-plane $\beta_{3}$ solutions for various thicknesses and crack depths. In Fig. 5(a), it is observed that $\beta_{3}$ is a monotonously increasing function of $B / W$, as expected. The bounding value of $\beta_{3}$ for each $a / W$ was close to the plane strain value $\not \beta_{1}$, and a relative thickness of $B / W=40$ was sufficient for $\beta_{3}$ to saturate to the bounding value, as shown in Fig. 5(b).

$\beta_{3}$ for the ASTM standard 3PB specimen [14], for which $B / W=0.5$ and $0.45 \leq a / W \leq 0.55$, was negative. This finding seemed to support the fact that $J_{\mathrm{c}}$ was not bounded in the case of increasing $B / W$ for 3 PB specimens [1].

Interestingly, in Fig. 5(b), $\beta_{3}$ was not always a monotonously increasing function of $a / W$, as observed for the thin specimens of $B / W=0.1,0.25$ and 0.5 . For example, $\beta_{3}$ for $B / W=0.1$ was a monotonously decreasing function of $a / W$ and thus might lead to the incorrect conclusion that deep cracks lose the out-of-plane crack-tip constraint. However, by normalizing $T_{33}$ in terms of $T_{33}(N)^{1 / 2} / K_{0}$ ( $W$ was constant for all cases in this study) as shown in Fig. 6, it is clearly seen that $T_{33}$ increased monotonously as $a / W$ increased for all $B / W \mathrm{~s}$, which means that the out-of-plane crack-tip constraint level was strengthened due to the increase in crack depth, although the increase rate was smaller than $a^{1 / 2}$.

\section{Discussion}

In addition to the mid-plane $T$-stresses, the variations of the $\beta_{1}$ and $\beta_{3}$ solutions in the thickness direction were also plotted for various thicknesses for $a / W=0.5$ in Fig. 7 and 8 , respectively. Note that the mid-side node values were omitted in this figure. As observed in the left part of Fig. 7, the in-plane $\beta_{1}$ distributions changed little overall compared with the mid-plane value in the range of $x_{3} /(B / 2)=0$ to 0.8 . Specifically, these differences were in the range of 4.1 to $15.3 \%$. The differences were less than $5 \%$ if $x_{3} /(B / 2)$ was in the range of 0 to 0.5 , regardless of $B / W$.

On the other hand, the out-of-plane $\beta_{3}$ distributions in Fig. 8 showed a visible decrease in the thickness direction, considering that the ordinate of this figure ranges from -14 to 2 . However, the rate of decrease became small as $B / W$ increased, as is clear for the case of $B / W=40$. Note that both $T_{11}$ and $T_{33}$ diverged significantly in the vicinity of the free surface $\left(x_{3} /(B / 2)=0.8\right.$ to 1.0$)$ because $\varepsilon_{3}$ tends to be singular near the free surface and is not well calculated using FEA [3, 4]. Thus, the $T$ stresses near the free surface calculated by the present FEA method are known to be unreliable [12] and require further study. 


\section{Summary}

In the present study, the $T$-stress solutions for $3 \mathrm{~PB}$ specimens with a wide range of the crack depth-to-width ratio ( $a / W=0.2$ to 0.8$)$ and the specimen thickness-to-width ratio $(B / W=0.1$ to 40 ) were calculated using 3D elastic FEA. The results showed that 3D $T_{11}$ at the specimen mid-plane tended to deviate from the $2 \mathrm{D} T_{11}$ as $B / W$ increased, with the deviation saturating for $B / W \geq 2$. The mid-plane 3D $T_{11}$ between cases of $B / W=0.1$ and 40 was large as $54 \%$ for $a / W=0.2$ and suggested that the $3 \mathrm{D}$ effects should be properly considered for cases of short crack length, especially when $T_{11}$ is negative. The mid-plane $T_{33}$ increased with $B / W$ and was close to the plane strain value $T_{11}$ for $B / W \geq 2$.

\section{Acknowledgments}

This work was supported in part by JSPS KAKENHI Grant Number 24561038. Their support is greatly appreciated.

\section{Appendix}




\section{List of figures}

Fig. 1 Three-dimensional coordinate system for the region along the crack front

Fig. 2. Sketch of the loads and geometry of the 3 PB specimens

Fig. 3. Typical finite element model of a 3PB specimen $(W=25 \mathrm{~mm}, S / W=4, a / W=0.5, B / W=0.5)$

Fig. 4 Normalized $T_{11}$ solutions $\left(\beta_{1}\right)$ at the specimen mid-plane for 3PB specimens ( $\left.\nu=0.3\right)$

Fig. 5 Normalized $T_{33}$ solutions $\left(\beta_{3}\right)$ at the specimen mid-plane for 3PB specimens $(v=0.3)$

Fig. 6 Normalized $T_{33}$ solutions $\left(T_{33}(N V)^{1 / 2} / K_{0}\right)$ at the specimen mid-plane for $3 \mathrm{~PB}$ specimens $(\nu=0.3)$

Fig. 7 Variations of $\beta_{1}$ in the thickness direction along the crack front for various thicknesses when $a / W=0.5(\nu=0.3)$

Fig. 8 Variations of $\beta_{3}$ in the thickness direction along the crack front for various thicknesses when $a / W=0.5(\nu=0.3)$

\section{References}

[1] Rathbun HJ, Odette GR, Yamamoto T, Lucas GE. Influence of statistical and constraint loss size effects on cleavage fracture toughness in the transition-A single variable experiment and database. Engineering Fracture Mechanics. 2006;73:134-58.

[2] Meshii T, Lu K, Takamura R. A failure criterion to explain the test specimen thickness effect on fracture toughness in the transition temperature region. Engineering Fracture Mechanics. 2013;104: 184197.

[3] Nakamura T, Parks DM. Determinationof elastic $T$-stress along three-dimensional crack fronts using an interaction integral. International Journal of Solids and Structures. 1992;29(13):1597-1611.

[4] Fernández-Canteli A, Giner E, Fernández-Sáez J, Fernández- Zúñiga. A unified analysis of the inplane and out-of-plane constraints in 3-D linear elastic fracture toughness, Proceedings of the $19^{\text {th }}$ European Conference on Fracture. Kazan, Russia. 2012; p.1-8.

[5] Leevers PS, Radon JC. Inherent stress biaxiality in various fracture specimen geometries. International Journal of Fracture. 1982;19:311-325.

[6] Kfouri AP. Some evaluations of the elastic T-term using Eshelby's method. International Journal of Fracture. 1986;30(4):301-315. 
[7] Cardew GE, Goldthorpe MR, Howard IC, Kfouri AP. On the Elastic T-term. In: Bibly BA, Miller KJ, Willis JR, editors. Fundamentals of Deformation and Fracture. Cambridge: Cambridge University Press; 1984. p. $465-476$.

[8] Fett T. T-stresses in rectangular plates and circular disks. Engineering Fracture Mechanics. 1998;60(56):631-652.

[9] Yang B, Ravi-Chandar K. Evaluation of elastic $T$-stress by the stress difference method. Engineering Fracture Mechanics. 1999;64(5):589-605.

[10] Sherry AH, Moran B, Nakamura T. Compendium of $T$-stress solutions for two and three dimensional cracked geometries. Fatigue and Fracture of Engineering Materials and Structures. 1995;18:141-155.

[11] Williams ML. On the stress distribution at the base of a stationary crack. Journal of Applied Mechanics. 1957;24:111-114.

[12] Qu J, Wang X. Solutions of $T$-stresses for quarter-elliptical corner cracks in finite thickness plates subject to tension and bending. International Journal of Pressure Vessels and Piping. 2006;83(8):593-606.

[13] Gullerud A, Koppenhoefer K, Roy Y, RoyChowdhury S, Walters M, Bichon B, et al. WARP3D Release 15 Manual. Civil Engineering, Report No UIUCENG-95-2012, University of Illinois at UrbanaChampaign. 2004.

[14] ASTM. E1921-10 Standard test method for determination of reference temperature, $T_{0}$, for ferritic steels in the transition range. Annual Book of ASTM Standards. Philadelphia PA: American Society for Testing and Materials; 2010. 
Table 1. Normalized $T_{11}$ solutions $\left(\beta_{1}\right)$ at the specimen mid-plane for 3PB specimens $(v=0.3)$.

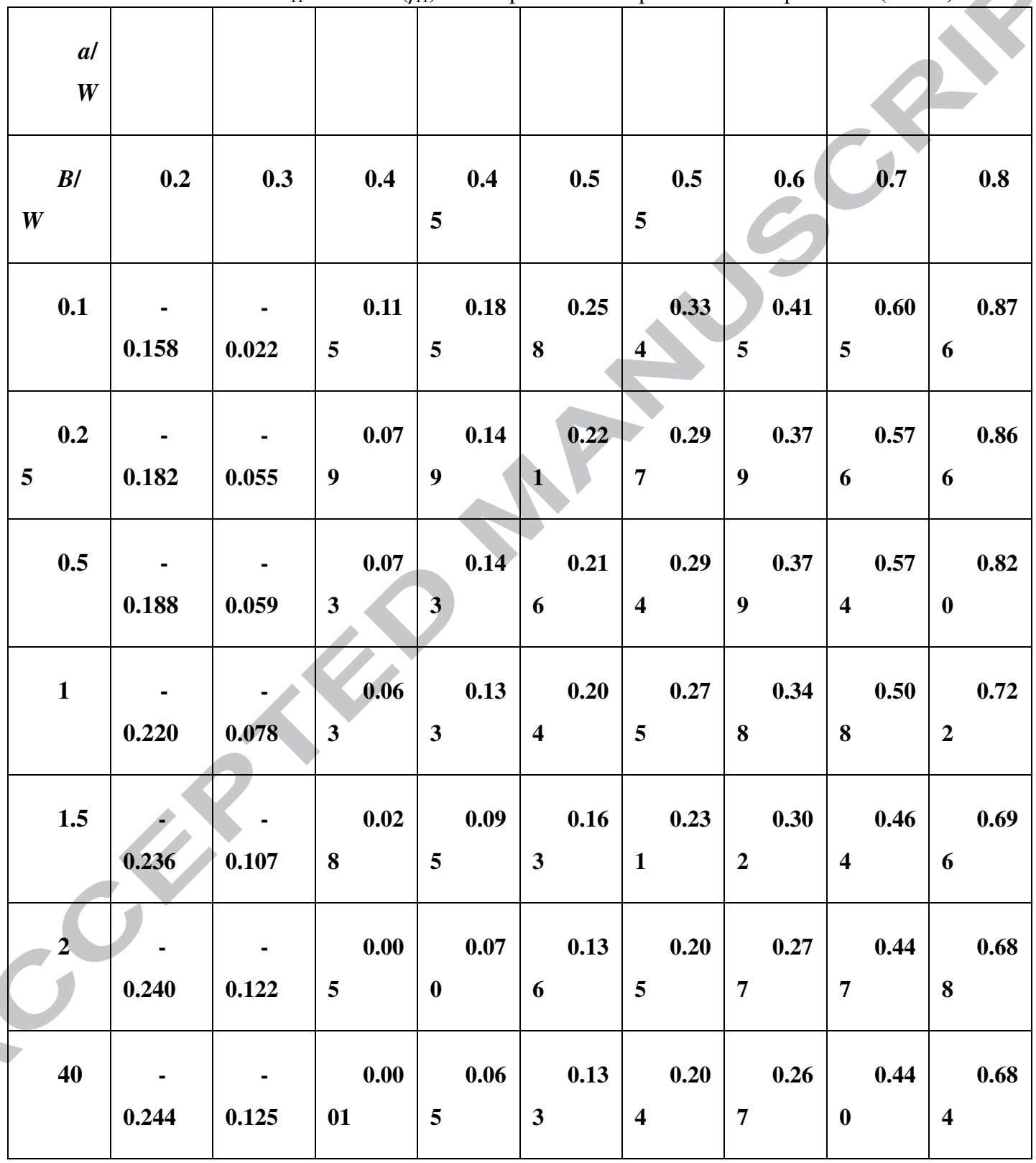


Table 2 Normalized $T_{33}$ solutions $\left(\beta_{3}\right)$ at the specimen mid-plane for $3 \mathrm{~PB}$ specimens $(v=0.3)$

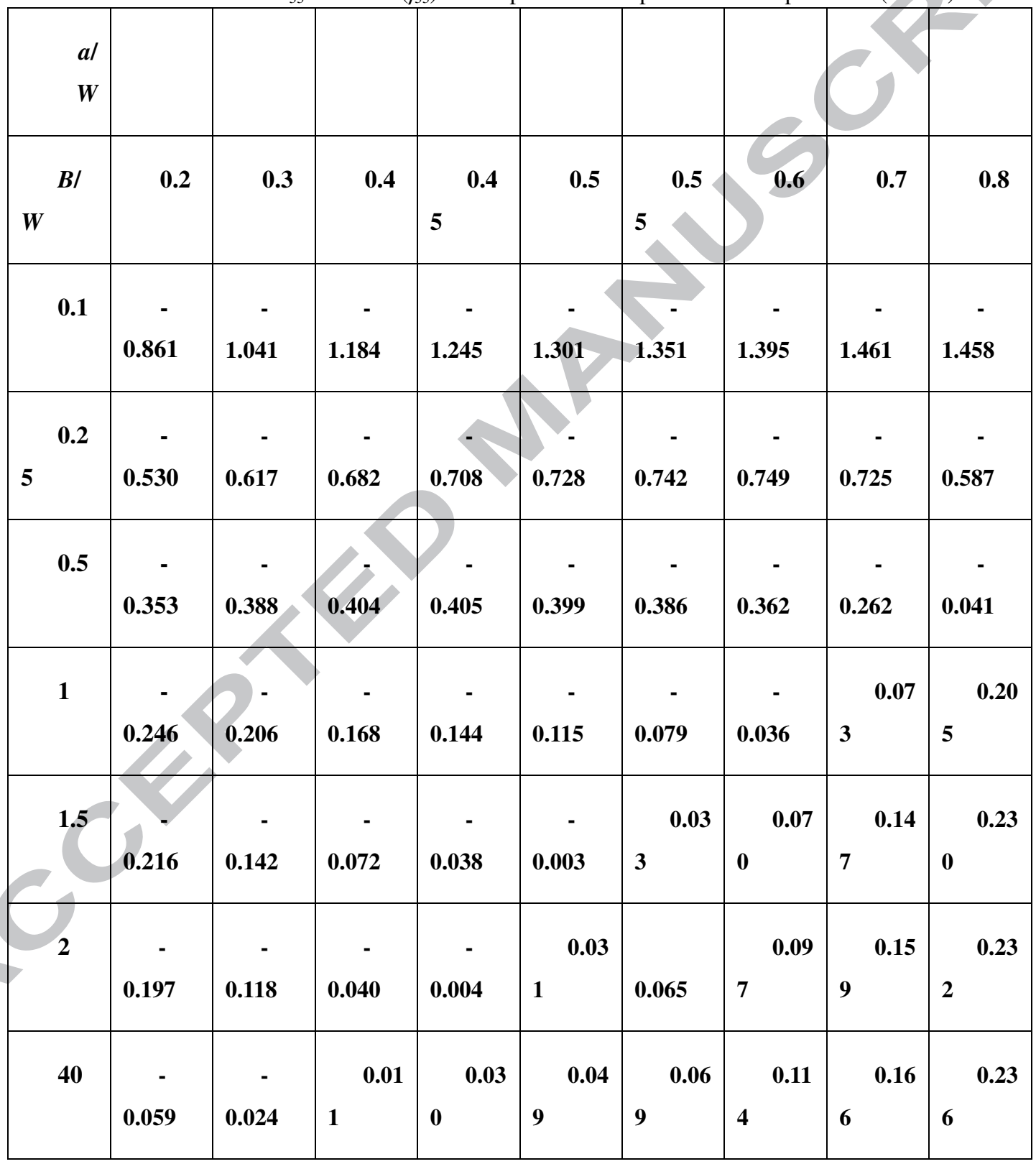




\section{ACCEPTED MANUSCRIPT}

Table A.1 Summary of the generated mesh $\left(W=25 \mathrm{~mm}, S / W=4, R_{\mathrm{s}}=0.4 \mathrm{~mm}\right)$

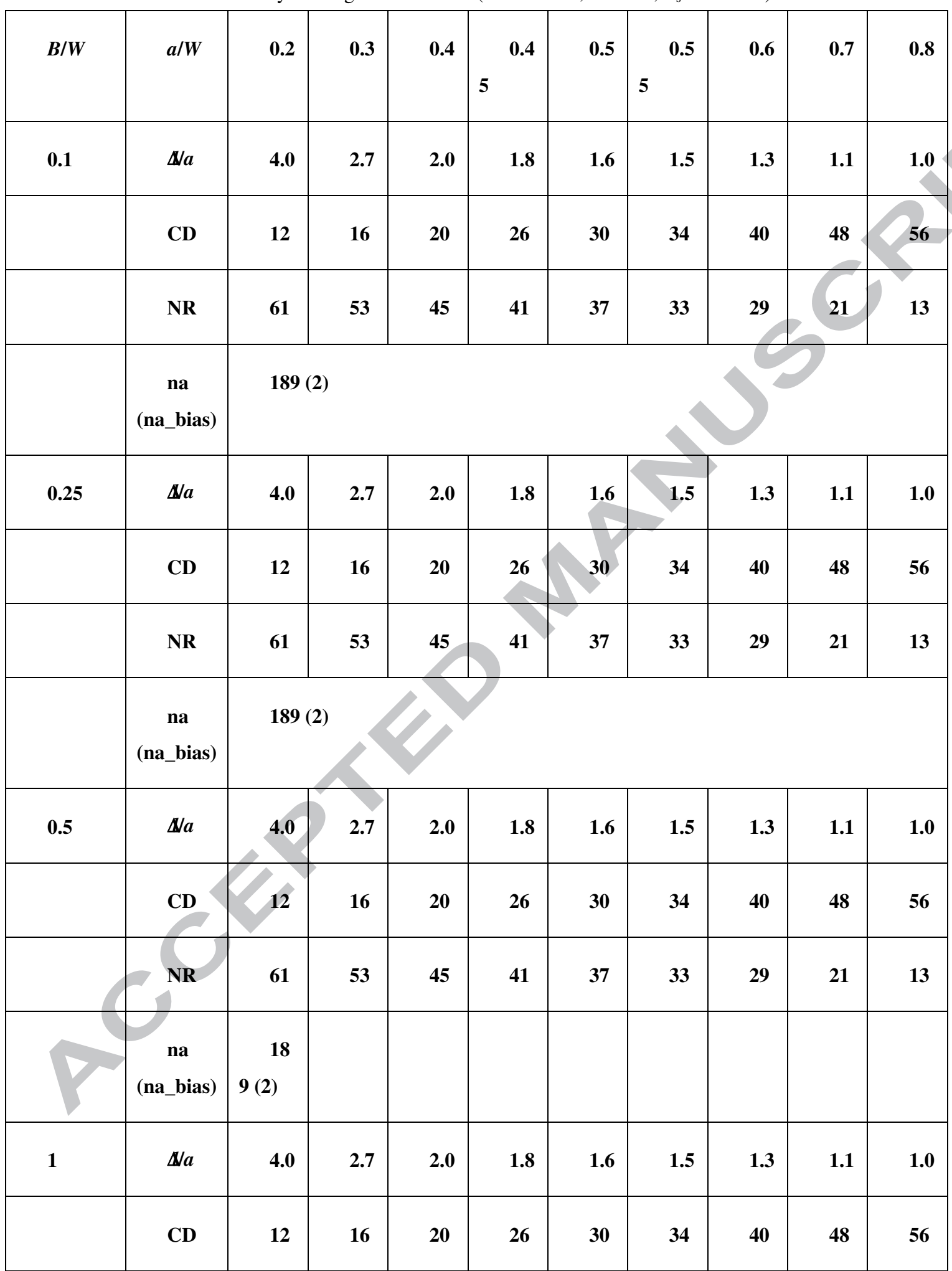




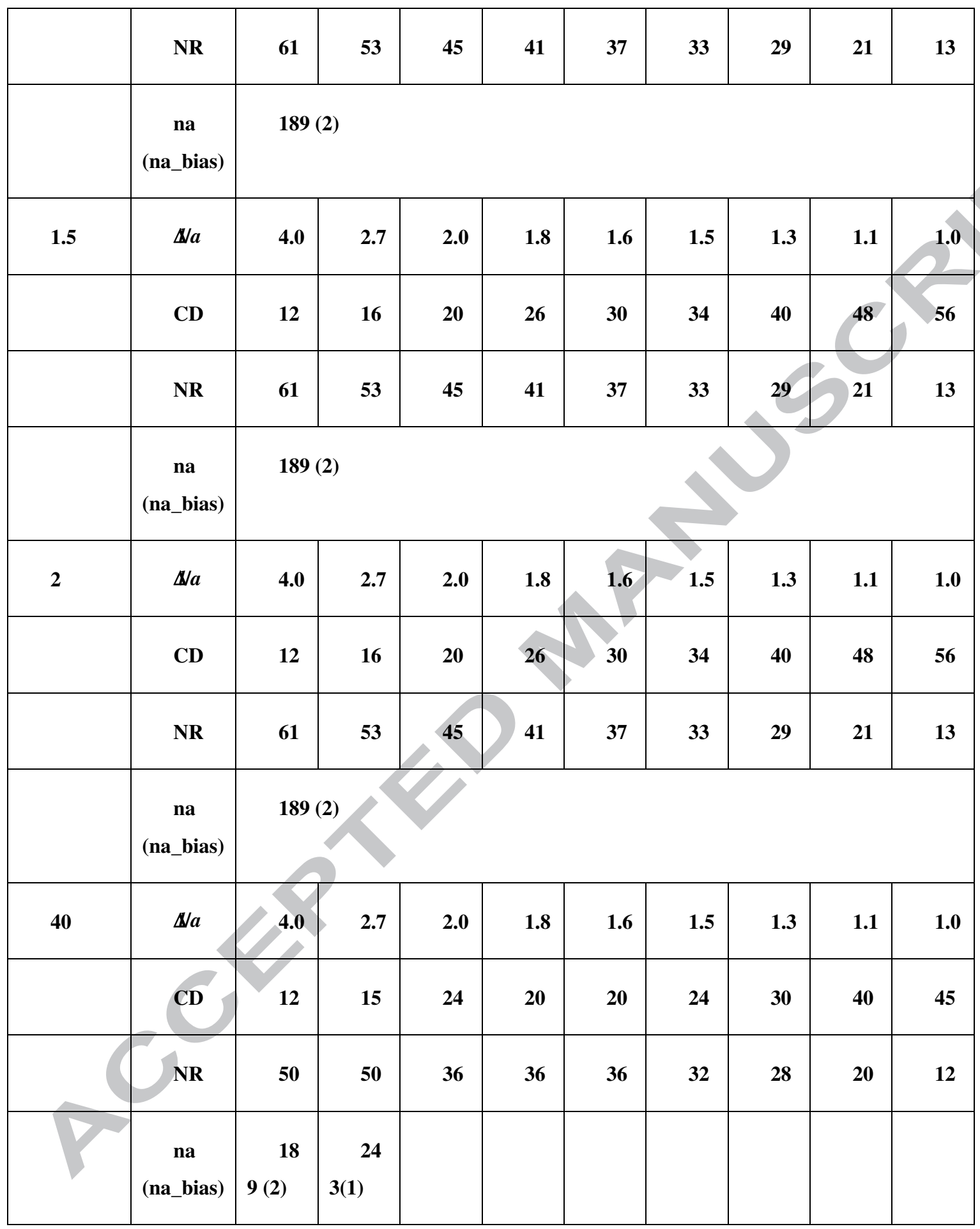




\section{Highlights}

- $T$-stress solutions 3PB specimens with various crack depths and thicknesses were obtained.

- Mid-plane $T_{11}$ and $T_{33}$ were reported for 3PB specimens with $a / W=0.2 \sim 0.8$ and $B / W=0.1 \sim 40$.

- $T_{11}$ showed 3D effect, and approached 2D plane strain solutions for large thickness.

- $T_{33}$ increased with thickness, and saturated to $V_{1}$ for $B / W \geq 2$.

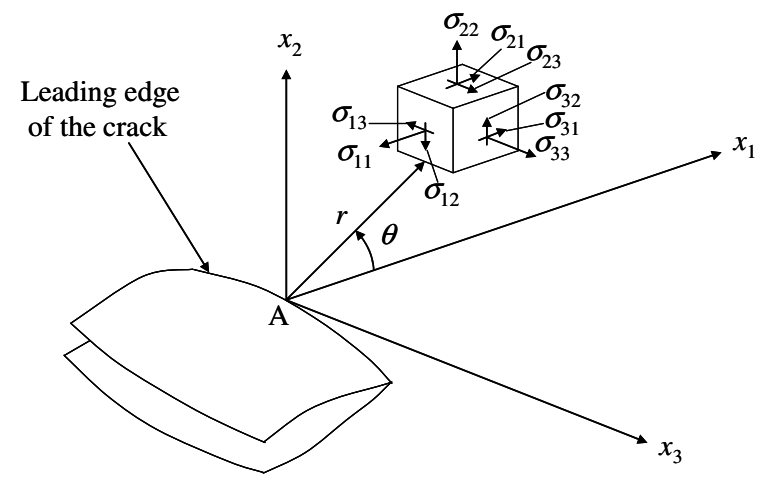

Fig. 1 Three-dimensional coordinate system for the region along the crack front 


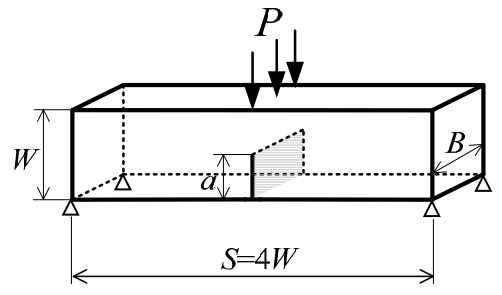

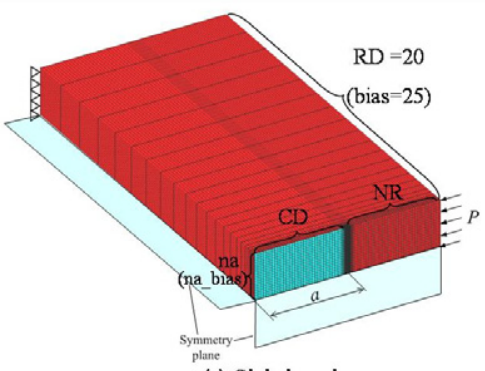

(a) Global mesh

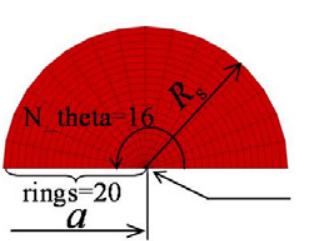

(b) Detail of crack tip for elastic analysis

Fig. 3 Typical finite element model of a 3PB specimen $(W=25 \mathrm{~mm}, S / W=4, a / W=0.5, B / W=0.5)$

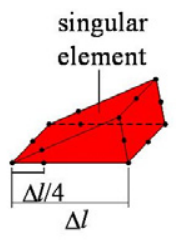

lement $\Delta l$ 


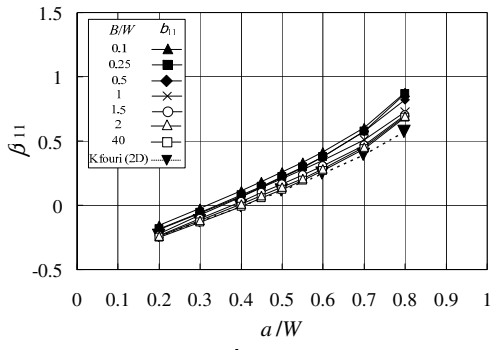

(a) $b_{11}$ vs $a / W$

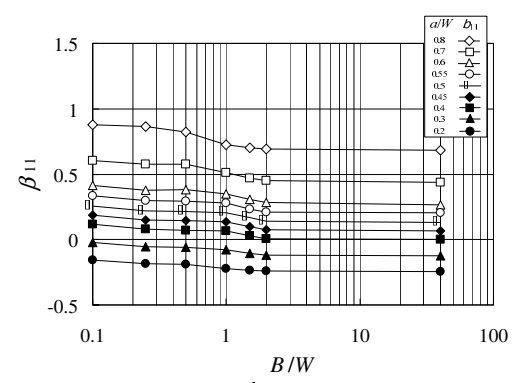

(b) $b_{11}$ vs $B / W$

Fig. 4 Normalized $T_{11}$ solutions $\left(\beta_{11}\right)$ at the specimen mid-plane for 3PB specimens $(\nu=0.3)$

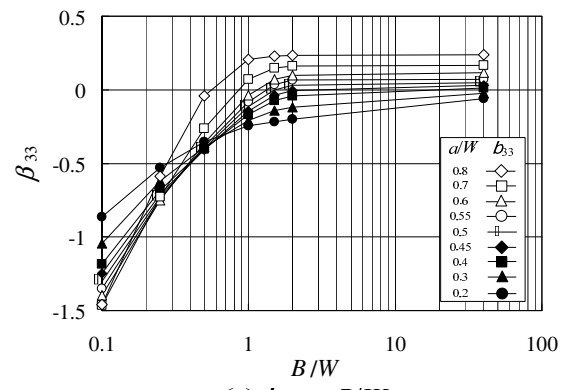

(a) $b_{33}$ vs $B / W$

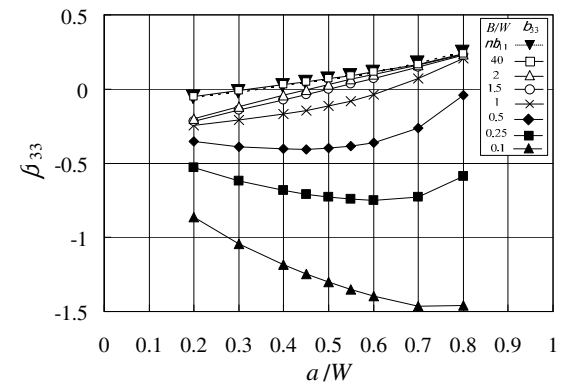

(b) $b_{33}$ vs $a / W$

Fig. 5 Normalized $T_{33}$ solutions $\left(\beta_{33}\right)$ at the specimen mid-plane for $3 \mathrm{~PB}$ specimens $(v=0.3)$ 


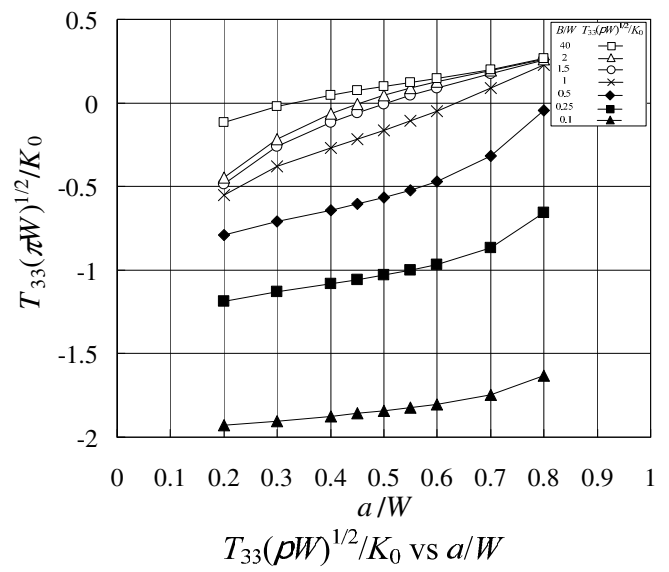

Fig. 6 Normalized $T_{33}$ solutions $\left(T_{33}(\pi W)^{1 / 2} / K_{0}\right)$ at the specimen mid-plane for 3PB specimens $(\nu=0.3)$

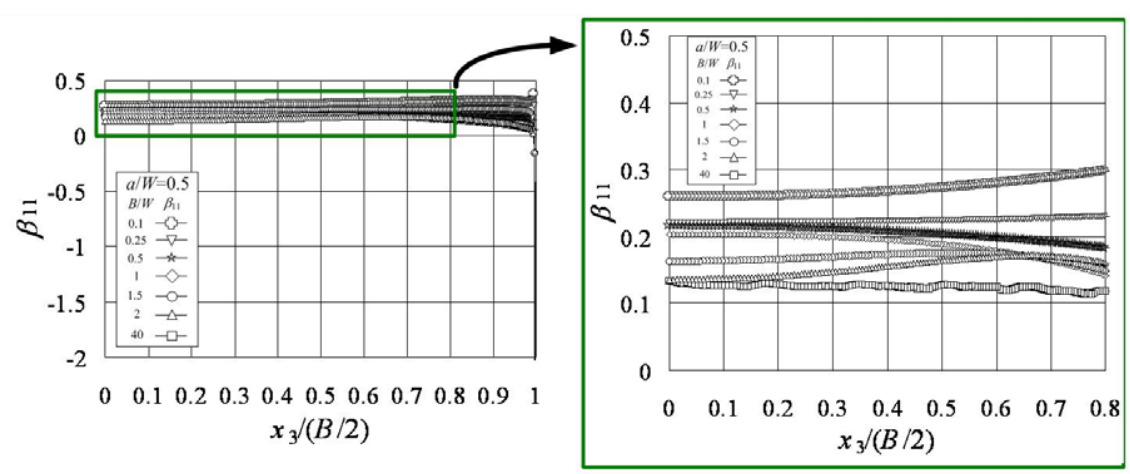

Fig. 7 Variations of $\beta_{11}$ in the thickness direction along the crack front for various thicknesses when $a / W=0.5(\nu=0.3)$ 


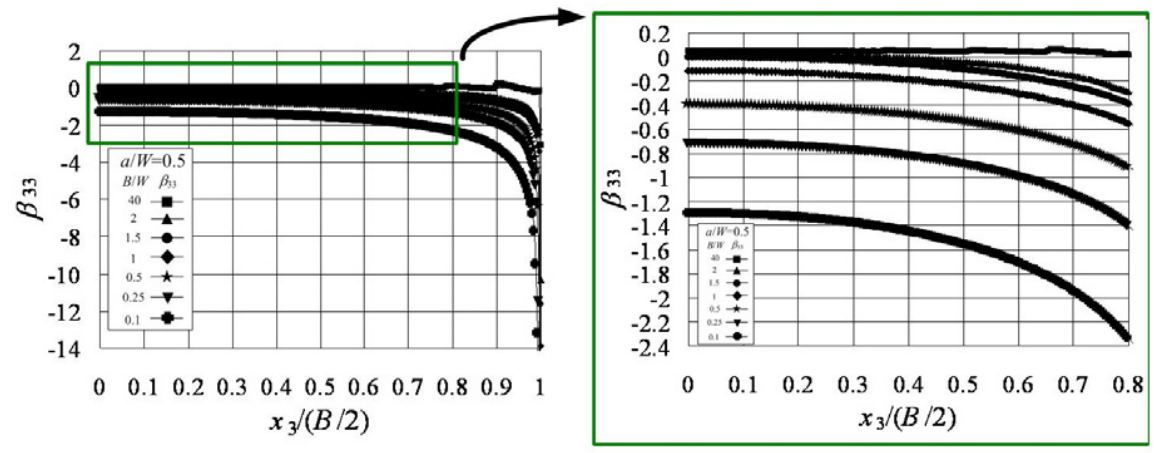

Fig. 8 Variations of $\beta_{33}$ in the thickness direction along the crack front for various thicknesses when $a / W=0.5(v=0.3)$ 